\title{
New treatment options for ALK+ advanced non- small-cell lung cancer: critical appraisal of ceritinib
}

This article was published in the following Dove Press journal:

Therapeutics and Clinical Risk Management

5 May 2016

Number of times this article has been viewed

\section{Sacha I Rothschild}

Department of Internal Medicine, Medical Oncology, University Hospital Basel, Basel, Switzerland
Correspondence: Sacha I Rothschild Department of Internal Medicine, Medical Oncology, University Hospital Basel, Petersgraben 4, 403I Basel, Switzerland Tel +4I 6 I 2655074

Fax +4I 6I 2655316

Email sacha.rothschild@usb.ch
Abstract: Rearrangements in ALK gene and EML4 gene were first described in 2007. This genomic aberration is found in about $2 \%-8 \%$ of non-small-cell lung cancer (NSCLC) patients. Crizotinib was the first ALK tyrosine kinase inhibitor licensed for the treatment of metastatic ALK-positive NSCLC based on a randomized Phase III trial. Despite the initial treatment response of crizotinib, disease progression inevitably develops after approximately 10 months of therapy. Different resistance mechanisms have recently been described. One relevant mechanism of resistance is the development of mutations in ALK. Novel ALK tyrosine kinase inhibitors have been developed to overcome these mutations. Ceritinib is an oral second-generation ALK inhibitor showing clinical activity not only in crizotinib-resistant ALK-positive NSCLC but also in treatment-naïve ALK-positive disease. In this paper, preclinical and clinical data of ceritinib are reviewed, and its role in the clinical setting is put into perspective.

Keywords: lung cancer, ALK, ceritinib, crizotinib

\section{Introduction to the targeted treatment of ALK- positive advanced non-small-cell lung cancer}

Non-small-cell lung cancer (NSCLC) remains the leading cause of cancer-related deaths in the USA and European countries and claims more lives per year than all other major cancer types combined. ${ }^{1}$ Two-third of patients are diagnosed with incurable metastatic disease in which the median overall survival (OS) with standard chemotherapy is approximately $12-14$ months. In recent years, an improved understanding of the molecular and genetic background of lung cancers has changed treatment paradigms for these patients. With the detection of activating mutation in EGFR, ${ }^{2,3}$ rearrangements of ALK with EML4 4 and others, a more personalized and targeted therapeutic approach is the standard of care for a subset of patients with lung adenocarcinoma. ALK is a transmembrane tyrosine kinase receptor that is expressed in neural tissue, the small intestine, and the testes, and plays a crucial role in the development of the central nervous system (CNS). ${ }^{5}$ The ALK receptor is activated after ligand binding to the extracellular receptor domain and dimerization. ${ }^{4}$ The EML4-ALK fusion gene arises from an inversion on the short arm of chromosome 2. ${ }^{4}$ Several variants of EML4ALK have been described that encode the same cytoplasmic tyrosine kinase domain of ALK with different truncations of EML4. ${ }^{6,7}$ Aberrant ALK activation leads to an activation of multiple downstream signaling pathways, primarily the PI3K/mTOR and RAS/RAF/MAPK cascade. ${ }^{8-10}$

The EML4-ALK rearrangement in patients with NSCLC is a relatively rare event, and it is present in approximately $2 \%-8 \%$ of NSCLC patients. ${ }^{4,7,11}$ It is most commonly seen in younger patients with adenocarcinoma histology with never- or light-smoking 
history. ALK rearrangements and other oncogenic drivers, such as mutant EGFR and oncogenic RAS, are generally mutually exclusive, consistent with the notion that ALK rearrangement defines a unique molecular subset of NSCLC. ${ }^{12}$ In patients with adenocarcinoma lacking EGFR and KRAS mutations, the prevalence of EML4-ALK translocation could be as high as $42.8 \% .^{13}$ In these patients, ALK rearrangements serve as a key and strong oncogenic driver for NSCLC and represent a critical therapeutic target susceptible to targeted ALK inhibition. ${ }^{4,14}$

The ALK tyrosine kinase inhibitor (ALK-TKI) crizotinib was the first targeted drug showing clinical activity in this patient population, with response rates similar to what has been seen in EGFR-mutant NSCLC with EGFR-TKIs. ${ }^{15}$ The PROFILE 1007 trial was the first randomized Phase III trial for ALK-positive patients. ${ }^{16}$ In total, 347 patients with ALKpositive NSCLC previously treated with chemotherapy were randomized between crizotinib or chemotherapy (pemetrexed or docetaxel). The overall response rate (ORR) of crizotinib was $65 \%$ compared to $20 \%$ in the chemotherapy group. The primary end point of progression-free survival (PFS) was prolonged from 3 months with chemotherapy to 7.7 months for patients treated with crizotinib (hazard ratio [HR]: 0.49, 95\% confidence interval [CI]: 0.37-0.64). The PROFILE 1014 trial included 343 chemotherapy-naïve ALK-positive patients randomized to crizotinib or platinum-based chemotherapy. ${ }^{17}$ PFS was significantly longer with crizotinib (10.9 vs 7 months, HR: $0.45,95 \%$ CI: 0.35-0.60). Based on these data, crizotinib has been approved in several countries including the USA, most European countries, and Switzerland and is the recommended first-line therapy for ALK-positive patients. ${ }^{18}$ While crizotinib has impressive activity in patients with ALK-rearranged NSCLC, these cancers invariably progress, typically in less than 1 year, because of the development of resistance to crizotinib. Therefore, the development of ALKTKIs with clinical activity against ALK-positive NSCLC resistant to crizotinib is crucial.

However, not all initially ALK-positive NSCLC remain dependent on the ALK signaling pathway in the situation of acquired resistance to crizotinib. Resistance develops through a variety of mechanisms. ALK-dependent mechanisms include secondary mutation of ALK or EML4-ALK gene amplification. In a first report on acquired resistance, two de novo point mutations in EML4-ALK (L1196M and C1156Y) have been described. ${ }^{19}$ The L1196M mutation added a new bulky residue in the ATP-binding domain, sterically hindering TKI binding, thus, acting as a gatekeeper mutation, similar to the T790M mutation in EGFR-mutant
NSCLC. Recently, several other secondary mutations have been described. ${ }^{20,21}$ Some patients were shown to have $A L K$ gene copy number gain. ${ }^{22}$ However, there exist also ALKindependent resistance mechanisms, mainly including the activation of bypassing signaling pathways. Some patients loose ALK positivity by FISH (fluorescence in situ hybridization), and may exhibit increased EGFR signaling or activation of KRAS. ${ }^{22}$ Another study also showed the activation of KIT. ${ }^{23}$ In some patients, resistance to crizotinib remained unexplained. ${ }^{22,23}$

\section{Preclinical data, pharmacokinetics, and pharmacodynamics}

Ceritinib (5-Chloro-N2-[2-isopropoxy-5-methyl-4-(4piperidinyl) phenyl]-N4-[2-(isopropylsulfonyl) phenyl]-2,4pyrimidinediamine) is an ATP-competitive TKI with a high selectivity for the ALK tyrosine kinase domain. ${ }^{24}$ Ceritinib has shown higher potency in inhibiting ALK than crizotinib in vitro and in animal experiments. ${ }^{25}$ The higher enzymatic inhibitory potency of ceritinib is explained by differences in the chemical structure compared to crizotinib. Mainly, the chlorine in the fifth position of the pyrimidine ring enhances interactions of ceritinib with the mutant residues at the gatekeeper position of the ALK kinase domain. ${ }^{25}$ Moreover, ceritinib possesses an isopropyl group that interacts with mutant ALK as well as a sulfonyl group interacting with the K1150 amino acid of ALK. ${ }^{26}$ In preclinical experiments, ceritinib exhibited more potent activity than crizotinib in terms of ALK phosphorylation suppression and cell growth inhibition. ${ }^{25}$ The half-maximal inhibitory concentration $\left(\mathrm{IC}_{50}\right)$ of ceritinib is approximately $150 \mathrm{pmol} / \mathrm{L}$, which is 20 -fold lower than that of crizotinib. ${ }^{25}$ In addition to ALK, ceritinib also inhibits other tyrosine kinase receptors, including the IGF1 and insulin receptors. ${ }^{27,28}$ Furthermore, ceritinib has shown activity toward the two most common EML4-ALK resistance mutations under crizotinib, L1196M and G1269A. ${ }^{25}$ Moreover, ceritinib has also shown activity against ALK-positive lung cancer cells derived from patients progressing under alectinib, another second-generation ALKTKI. ${ }^{29}$ However, C1156Y, G1202R, 1151T-ins, L1152R, and F1174C secondary ALK mutations were associated with resistance to ceritinib. ${ }^{25}$ In patient-derived models of acquired ceritinib resistance, activation of the MEK and the SRC signaling pathway have been identified as potential resistance mechanisms. ${ }^{27,30}$

Ceritinib is taken orally once daily. Ceritinib is slowly absorbed, with median peak plasma concentration occurring at approximately 4-6 hours in patients and at approximately 
6-8 hours in healthy subjects. Following peak concentration in the plasma $\left(C_{\max }\right)$, ceritinib concentrations declined in a monoexponential manner. The geometric mean, apparent terminal half-life ranged from 31 to 41 hours across the 400-750 mg dose groups in patients, and from 36 to 48 hours across the 450-750 $\mathrm{mg}$ dose groups in healthy subjects. Ceritinib is highly bound to plasma protein $(>94 \%)$ in all species. CYP3A4/5 is the major hepatic enzyme metabolizing ceritinib in a human in vitro system. Ceritinib is likely a P-gp, but not BCRP or MRP2 substrate. It does not inhibit P-gp, BCRP, or MRP2 up to a concentration of $1.5 \mu \mathrm{M}$ in vitro.

A food effect study conducted in healthy subjects showed that, following a single $500 \mathrm{mg}$ oral dose of ceritinib, the bioavailability of ceritinib is increased when the dose is given with a meal. ${ }^{31}$ Compared to the fasted condition, a low-fat meal (containing approximately 330 calories and $9 \mathrm{~g}$ of fat) increased $C_{\max }$ and area under the curve by $43 \%$ and $58 \%$, respectively, whereas a high-fat meal (containing approximately 1,000 calories and $58 \mathrm{~g}$ of fat) increased $C_{\max }$ and area under the curve by $41 \%$ and $73 \%$, respectively. In an ongoing open-label, multicenter, randomized, parallel design Phase I study, the systemic exposure and safety of ceritinib administered at 450 or $600 \mathrm{mg}$ with a daily low-fat meal vs $750 \mathrm{mg}$ daily in the fasted state will be determined following multiple oral daily dosing in patients with metastatic ALKpositive NSCLC. The primary objective of this study is to assess the steady-state pharmacokinetics of 450 or $600 \mathrm{mg}$ ceritinib taken with a low-fat meal as compared with that of $750 \mathrm{mg}$ ceritinib taken in the fasted state in patients with metastatic ALK-positive NSCLC. Secondary objectives will be to assess the safety and efficacy of ceritinib when administered at 450 or $600 \mathrm{mg}$ with a daily low-fat meal or $750 \mathrm{mg}$ daily in the fasted state.

\section{Clinical data}

In the ASCEND-1 trial, 59 patients with solid tumors carrying genetic alterations in ALK were treated with ceritinib. ${ }^{32}$ The dose was escalated from 50 to $750 \mathrm{mg}$ given orally once daily. The maximum tolerated dose was $750 \mathrm{mg}$ once daily. From this Phase I trial, efficacy data were reported from 114 patients being treated with at least $400 \mathrm{mg}$ ceritinib per day. ${ }^{32}$ The ORR in this heavily pretreated patient population was $58 \%$ (95\% CI: 48-67), with a median PFS of 7 months (95\% CI: 5.6-9.5) and a survival rate of 65\% after 1 year. About two-third of the study population was pretreated with crizotinib $(n=80)$. In this subset of patients, ORR was almost identical to the whole population, at 56\% (95\% CI: 45-67).
However, median PFS was substantially longer in crizotinibnaïve patients than in crizotinib-pretreated patients $(10.4$ vs 6.9 months). Interestingly, responses were reported in patients with untreated brain metastases occurring during crizotinib therapy. PFS for patients with brain metastases was not different (6.9 vs 7 months, $P=0.37$ ). In summary, this Phase I trial with an expansion cohort was able to show promising efficacy data of ceritinib in ALK-positive lung adenocarcinoma. Based on these results, the US Food and Drug Administration granted accelerated approval to ceritinib in April 2014.

An update of the expansion cohort of the ASCEND-1 trial with 246 ALK-positive NSCLC patients has been reported at the European Society for Medical Oncology (ESMO) conference $2014 .{ }^{33}$ Of these patients, 163 were pretreated with crizotinib and 5 of them have also been treated with alectinib. Median age of the cohort was 53 years, with a predominance (53.7\%) of female patients and never- or ex-smokers (97.6\%). Approximately, one-fourth of patients were pretreated with one, two, or more than three treatment lines. ORR was $61.8 \%$ (95\% CI: 55.4-67.9) in the whole population and 56.4\% (95\% CI: 48.5-64.2) in crizotinib-pretreated patients. Median PFS was 9 months (95\% CI: 6.9-11.0). In patients previously treated with another ALK-TKI, median PFS was 6.9 months (95\% CI: 5.6-8.7), whereas it was 18.4 months (95\% CI: 11.1 to not estimable) in ALK-TKI-naïve patients.

ASCEND-2 was a single-arm, open-label, multicenter Phase II study for patients with ALK-positive NSCLC previously treated with one to three prior lines of chemotherapy, of which one must have been a platinum-based regimen and crizotinib. ${ }^{34}$ As in the other ceritinib trial, patients with asymptomatic or neurologically stable brain metastases were eligible. This trial included 140 patients, the majority presented with brain metastases at study entry $(71.4 \%)$, of whom $72 \%$ had received prior brain radiation. In total, $22.1 \%$ of patients received more than one line of prior chemotherapy. Median duration of ceritinib therapy was 8.8 months. The ORR by investigator review was $84.9 \%$ (95\% CI: 30.5-47.2), $75.2 \%$ of patients had a decrease in tumor burden from baseline. Median PFS was 5.7 months (95\% CI: 5.4-7.6), and median OS was 14.9 months (95\% CI: 13.5 to not reached). At data cutoff, 72 patients were still alive, therefore final survival analysis is pending.

In the ASCEND-3 trial, 124 patients with ALK-positive NSCLC who had not received prior treatment with an ALKTKI were included. ${ }^{35}$ Almost all patients (98.4\%) have received at least one prior chemotherapy treatment. Patient with asymptomatic or neurologically stable brain metastases 
were eligible to enroll. ASCEND-3 was a single-arm, openlabel, Simon's two-stage Phase II study with ORR based on Response Evaluation Criteria in Solid Tumors ${ }^{36}$ as the primary end point. Patients were treated with ceritinib $750 \mathrm{mg}$ daily. The majority of patients were Asian (59.7\%). A total of $50(40.3 \%)$ patients presented with brain metastases at study entry, of whom 27 (54.0\%) patients had received prior brain radiation. One-fourth of patients were pretreated with three or more lines of chemotherapy. The median duration of ceritinib exposure was 8 months, with an ORR by investigator review of $63.7 \%$ (95\% CI: 54.6-72.2). Almost all patients $(93.9 \%)$ had a decrease in tumor burden from baseline. Responses were durable, with a median duration of response of 9.3 months (95\% CI: 9.1 to not reached due to ongoing responses at the time of analysis) and a median PFS of 11.1 months (95\% CI: 9.3 to not reached). The median OS has not been reached at the time of data cutoff (median duration of follow-up 8.3 months). OS after 1 year was estimated to be $81.5 \%$ (95\% CI: 64.8-90.8).

\section{Brain metastases}

Involvement of the CNS in NSCLC is frequent. The reported lifetime incidence of CNS metastases in ALK-positive NSCLC is approximately $35 \%-50 \% .{ }^{37} \mathrm{CNS}$ is the most common site of disease progression on crizotinib in patients with and without CNS metastases at baseline. Crizotinib shows comparable disease control rates for brain metastases and extracranial disease. However, the progression of preexisting or development of new intracranial lesions while receiving therapy was a common manifestation of acquired resistance to crizotinib. ${ }^{38}$ In the ASCEND-2 trial of 20 patients with an active target lesion in the brain at baseline, 45\% (95\% CI: 23.1-68.5) showed intracranial response and 80\% (95\% CI: 56.3-94.3) showed intracranial disease stabilization with ceritinib. ${ }^{34}$ In the ASCEND-3 trial, ten patients had investigator-assessed brain lesions selected as active target lesions at baseline. ${ }^{35}$ The response rate in the brain was $20.0 \%$ (95\% CI: 2.5-55.6), with an intracerebral disease control rate of $80.0 \%$ (95\% CI: 44.4-97.5).

\section{Quality of life}

The ASCEND-2 trial included evaluation of quality of life (QOL) with different questionnaires (QLQ-C30, LC-13, and LCSS). ${ }^{34}$ Global QOL score (QLQ-C30) was maintained during treatment, whereas symptom burden was improved with ceritinib.

In the ASCEND-3 trial, $82.1 \%$ of patients showed improvements in symptom burden from baseline when treated with ceritinib. ${ }^{35}$ Global QOL score assessed by
QLQ-C30 was maintained on treatment. However, patients initially showed worsening score at cycle 2, with improvement at later cycles. Therefore, it can be hypothesized that only patients responding to therapy and being treated for a longer time experience a benefit regarding QOL. In QOL assessment, mainly gastrointestinal (GI) problems (diarrhea, nausea, and vomiting) accounted for worsening rating of QOL.

\section{Safety and tolerability of ceritinib}

Ceritinib is associated with a manageable safety profile. The most common adverse events (AEs), regardless of study drug relationship (incidence $\geq 25 \%$ ), were diarrhea, nausea, vomiting, ALT increase, fatigue, abdominal pain, decreased appetite, AST increase, and constipation. ${ }^{31}$ Incidence of Grade 3 and 4 AEs, regardless of study drug relationship, was $<5 \%$ for all AEs except ALT increase (26.7\%), AST increase $(8.2 \%)$, diarrhea $(5.9 \%)$, hyperglycemia $(5.5 \%)$, lipase increase $(5.1 \%)$, and blood alkaline phosphatase increase $(5.1 \%) .{ }^{31}$ In daily clinical practice, the high rate of GI toxicity (nausea, vomiting, and diarrhea, being the most common AEs) is of major concern, as it negatively impacts patients QOL and may interfere with drug absorption.

In the Phase I trial, 59 patients have been included in the dose-finding cohort. ${ }^{32}$ The dose of $750 \mathrm{mg}$ taken once daily orally was defined as the maximum tolerated dose. Doselimiting toxicities that have been reported were diarrhea, vomiting, nausea, dehydration, elevated ALT, and hypophosphatemia. All dose-limiting toxicities resolved after treatment discontinuation. The same trial recruited a further 71 patients treated with $750 \mathrm{mg}$ in an expansion cohort. The most frequently reported side effects were nausea $(82 \%)$, diarrhea (75\%), vomiting (65\%), fatigue (47\%), and increased ALT levels (35\%). The rate of Grades 3 and 4 AEs was generally low, with transaminases elevation (21\%) and diarrhea (7\%) occurring most often. The rate of patients discontinuing treatment or reducing the dose of ceritinib was $6 \%$ and $51 \%$, respectively. Four patients were diagnosed with interstitial lung disease possibly related to therapy. All patients fully recovered after treatment discontinuation.

In the ASCEND-1 trial, median relative dose intensity was $82.8 \%$ (range: $30.2-100$ ) for ALK-positive NSCLC patients treated at the $750 \mathrm{mg}$ daily dose. ${ }^{33}$ In total, $75.2 \%$ of patients had at least one dose interruption, while $61.8 \%$ had one dose reduction. Furthermore, $19.2 \%$ and $6.7 \%$ of patients had two or three dose reductions, respectively. Incidence and type of AEs were comparable to previously reported safety results. Serious AEs (SAEs) were reported in 47.5\% of patients, and $12.5 \%$ patients had a SAEs suspected to be 
Table I Most common adverse events of ceritinib and crizotinib

\begin{tabular}{lll}
\hline & Ceritinib & Crizotinib \\
\hline Most common adverse events & Diarrhea, & Vision disorders \\
& constipation & Nausea, vomiting \\
& Nausea, vomiting & Diarrhea, \\
& Fatigue & constipation \\
& Hepatotoxicity & Edema \\
& Abdominal pain & Hepatotoxicity \\
& Rash & Reduced appetite \\
& Serum creatinine & Vertigo \\
& elevation & Neuropathy \\
& Achalasia & Interstitial \\
& & lung disease/ \\
Dose reduction (\%) & & Pneumonitis \\
Dose interruption (\%) & 62 & QT prolongation \\
Treatment discontinuation (\%) & $<10$ & I5 \\
\hline
\end{tabular}

drug-related. Again, the most common AEs were GI related (nausea, vomiting, and diarrhea).

Results from the early trial were confirmed in Phase II trials. In ASCEND-2 trial, most common AEs were nausea (81.4\%), diarrhea (80.0\%), and vomiting (62.9\%). ${ }^{34}$ In this trial, $41.4 \%$ of patients required dose adjustment or interruption. The ASCEND-3 trial showed similar findings with GI-related AEs reported most commonly: diarrhea (82.3\%), nausea $(74.2 \%)$, and vomiting (66.9\%). SAEs suspected to be study drug related were reported in ten (8.1\%) patients. Pericarditis was the only SAE suspected to be study drug related that was reported in three $(2.4 \%)$ patients. A total of 90 (72.6\%) patients required dose adjustment or interruption.

Based on these data, ceritinib seems to have a higher toxicity than crizotinib, and a substantial number of patients need dose reductions in the course of treatment. Table 1 summarizes the most common AEs of ceritinib and crizotinib from the package inserts.

\section{Conclusion, place in therapy}

The current standard of care for ALK-positive patients in the first-line setting is crizotinib, based on the results of the PROFILE 1014 trial. ${ }^{17}$ Ceritinib is approved for ALK-positive adenocarcinoma patients progressing on crizotinib. Ceritinib shows substantial activity in patients with brain metastases, a frequent site of disease progression in ALK-positive NSCLC. ${ }^{39}$ The occurrence of CNS relapse is one of the main concerns in ALK-positive patients treated with crizotinib. Therefore, ceritinib seems to be an active drug in patients progressing on crizotinib. ${ }^{34}$ Furthermore, ceritinib might be a valuable option in ALK-positive patients with brain metastases irrespective of previous therapies. On the other hand, ceritinib also shows high activity in ALK-TKI-naïve patients, with a median PFS of 11.1 months..$^{35}$ The question of the optimal sequence of different ALK-TKIs cannot be answered at the moment as there are no head-to-head comparisons. Currently, the only prospective randomized trial comparing two different ALK-TKIs is the ALEX trial investigating crizotinib and alectinib in the first-line setting (NCT02075840). The trial has finished recruitment at the end of 2015, and results are eagerly awaited. A similar trial in a Japanese population (J-ALEX, JapicCTI-132316) was stopped early after a recommendation of the independent data monitoring committee as the study met its primary endpoint. The study showed that patients lived significantly longer when treated with alectinib compared to crizotinib. ${ }^{40}$ Alectinib is a more selective and potent ALK inhibitor than crizotinib. Preclinical studies have demonstrated activity against the crizotinib-resistant ALK mutations L1196M, C1156Y, and F1174L. ${ }^{41}$ Other ALK inhibitors investigated in clinical trials include lorlatinib (PF-06463922), AP26113, ASP3026, TSR-011, and others. Lorlatinib for example is a highly potent, selective inhibitor of ALK and ROS1 with strong activity against all known ALK and ROS1 resistance mutations identified so far, including the highly resistant G1202R. There are several ongoing clinical trials with ceritinib. In two randomized Phase III trials, ceritinib is tested in comparison to platinum and pemetrexed in treatment-naïve ALK-positive NSCLC (NCT01828099) and in comparison to docetaxel in previously treated patients (NCT01828112). Furthermore, ongoing trials investigate ceritinib in combination with the anti-PD-1 inhibitor nivolumab (NCT02393625) and with the mTOR inhibitor everolimus (NCT02321501).

In conclusion, ceritinib is a highly active ALK-TKI that has its place in the treatment landscape of ALK-positive lung cancer patients. However, the optimal treatment sequence for these patients has not yet been defined. As ceritinib exhibits a substantial toxicity with mainly GI-related AEs, the question of optimal dosing and application to improve patients QOL while on treatment has to be critically evaluated.

\section{Disclosure}

SIR received honoraria from Novartis, Pfizer, and Roche for advisory boards and invited talks. The author reports no other conflicts of interest in this work.

\section{References}

1. Siegel RL, Miller KD, Jemal A. Cancer statistics. CA Cancer J Clin. 2015; 65(1):5-29.

2. Paez JG, Janne PA, Lee JC, et al. EGFR mutations in lung cancer: correlation with clinical response to gefitinib therapy. Science. 2004;304(5676) $1497-1500$ 
3. Lynch TJ, Bell DW, Sordella R, et al. Activating mutations in the epidermal growth factor receptor underlying responsiveness of non-small-cell lung cancer to gefitinib. N Engl J Med. 2004;350(21):2129-2139.

4. Soda M, Choi YL, Enomoto M, et al. Identification of the transforming EML4-ALK fusion gene in non-small-cell lung cancer. Nature. 2007; 448(7153):561-566.

5. Iwahara T, Fujimoto J, Wen D, et al. Molecular characterization of ALK, a receptor tyrosine kinase expressed specifically in the nervous system. Oncogene. 1997;14(4):439-449.

6. Choi YL, Takeuchi K, Soda M, et al. Identification of novel isoforms of the EML4-ALK transforming gene in non-small cell lung cancer. Cancer Res. 2008;68(13):4971-4976.

7. Takeuchi K, Choi YL, Togashi Y, et al. KIF5B-ALK, a novel fusion oncokinase identified by an immunohistochemistry-based diagnostic system for ALK-positive lung cancer. Clin Cancer Res. 2009;15(9): 3143-3149.

8. Soda M, TakadaS, TakeuchiK, etal. Amouse model forEML4-ALK-positive lung cancer. Proc Natl Acad Sci U S A. 2008;105(50):19893-19897.

9. Okamoto I, Nakagawa K. Echinoderm microtubule-associated proteinlike 4-anaplastic lymphoma kinase-targeted therapy for advanced non-small cell lung cancer: molecular and clinical aspects. Cancer Sci. 2012;103(8):1391-1396.

10. Sasaki T, Rodig SJ, Chirieac LR, Janne PA. The biology and treatment of EML4-ALK non-small cell lung cancer. Eur J Cancer. 2010;46(10): 1773-1780.

11. Scagliotti G, Stahel RA, Rosell R, Thatcher N, Soria JC. ALK translocation and crizotinib in non-small cell lung cancer: an evolving paradigm in oncology drug development. Eur J Cancer. 2012;48(7): 961-973.

12. Gainor JF, Varghese AM, Ou S-HI, et al. ALK rearrangements are mutually exclusive with mutations in EGFR or KRAS: an analysis of 1,683 patients with non-small cell lung cancer. Clin Cancer Res. 2013; 19(15):4273-4281.

13. Zhang X, Zhang S, Yang X, et al. Fusion of EML4 and ALK is associated with development of lung adenocarcinomas lacking EGFR and KRAS mutations and is correlated with ALK expression. Mol Cancer. 2010;9:188.

14. Kwak EL, Bang YJ, Camidge DR, et al. Anaplastic lymphoma kinase inhibition in non-small-cell lung cancer. $N$ Engl J Med. 2010; 363(18):1693-1703.

15. Rothschild SI, Gautschi O. Crizotinib in the treatment of non-small-cell lung cancer. Clin Lung Cancer. 2013;14(5):473-480.

16. Shaw AT, Kim DW, Nakagawa K, et al. Phase III study of crizotinib versus pemetrexed or docetaxel chemotherapy in patients with advanced ALK-positive non-small cell lung cancer (NSCLC) (PROFILE 1007). Ann Oncol. 2012;23(Suppl 9):LBA1_PR.

17. Solomon BJ, Mok T, Kim D-W, et al. First-line crizotinib versus chemotherapy in ALK-positive lung cancer. N Engl J Med. 2014;371(23): 2167-2177.

18. National Comprehensive Cancer Network. NCCN Clinical Practice Guidelines in Oncology-Non-Small Cell Lung Cancer. Fort Washington, PA: NCCN; 2015.

19. Choi YL, Soda M, Yamashita Y, et al. EML4-ALK mutations in lung cancer that confer resistance to ALK inhibitors. N Engl J Med. 2010; 363(18):1734-1739

20. Sasaki T, Koivunen J, Ogino A, et al. A novel ALK secondary mutation and EGFR signaling cause resistance to ALK kinase inhibitors. Cancer Res. 2011;71(18):6051-6060.

21. Heuckmann JM, Hölzel M, Sos ML, et al. ALK mutations conferring differential resistance to structurally diverse ALK inhibitors. Clin Cancer Res. 2011;17(23):7394-7401.

22. Doebele RC, Pilling AB, Aisner DL, et al. Mechanisms of resistance to crizotinib in patients with ALK gene rearranged non-small cell lung cancer. Clin Cancer Res. 2012;18(5):1472-1482.
23. Katayama R, Shaw AT, Khan TM, et al. Mechanisms of acquired crizotinib resistance in ALK-rearranged lung cancers. Sci Transl Med. 2012;4(120):120ra17.

24. Marsilje TH, Pei W, Chen B, et al. Synthesis, structure-activity relationships, and in vivo efficacy of the novel potent and selective anaplastic lymphoma kinase (ALK) inhibitor 5-chloro-N2-(2-isopropoxy-5-methyl4-(piperidin-4-yl)phenyl)-N4-(2-(isopropylsulfonyl)phenyl)pyrimidine2,4-diam. J Med Chem. 2013;56(14):5675-5690.

25. Friboulet L, Li N, Katayama R, et al. The ALK inhibitor ceritinib overcomes crizotinib resistance in non-small cell lung cancer. Cancer Discov. 2014;4(6):662-673

26. Ni Z, Zhang T-C. Computationally unraveling how ceritinib overcomes drug-resistance mutations in ALK-rearranged lung cancer. J Mol Model. 2015;21(7):175.

27. Cooper MR, Chim H, Chan H, Durand C. Ceritinib: a new tyrosine kinase inhibitor for non-small-cell lung cancer. Ann Pharmacother. 2015;49(1): 107-112.

28. Katayama R, Lovly CM, Shaw AT. Therapeutic targeting of anaplastic lymphoma kinase in lung cancer: a paradigm for precision cancer medicine. Clin Cancer Res. 2015;21(10):2227-2235.

29. Katayama R, Friboulet L, Koike S, et al. Two novel ALK mutations mediate acquired resistance to the next-generation ALK inhibitor alectinib. Clin Cancer Res. 2014;20(22):5686-5696.

30. Sequist LV, Gettinger S, Senzer NN, et al. Activity of IPI-504, a novel heat-shock protein 90 inhibitor, in patients with molecularly defined non-small-cell lung cancer. J Clin Oncol. 2010;28(33):4953-4960.

31. Zykadia ${ }^{\circledR}$ (ceritinib) capsules [prescribing information]. Novartis Pharma Switzerland; 2015

32. Shaw AT, Kim D-W, Mehra R, et al. Ceritinib in ALK-rearranged non-small-cell lung cancer. N Engl J Med. 2014;370(13):1189-1197.

33. Felip E, Kim D-W, Mehra R, et al. Efficacy and safety of ceritinib in patients (pts) with advanced anaplastic lymphoma kinase (ALK-) rearranged $(\mathrm{ALK}+)$ non-small cell lung cancer (NSCLC): an update of ASCEND-1. Ann Oncol. 2014;25(Suppl 4):1295P.

34. Mok T, Spigel D, Felip E, et al. ASCEND-2: a single-arm, open-label, multicenter phase 2 study of ceritinib in adult patients (pts) with ALK-rearranged (ALK+) non-small cell lung cancer (NSCLC) previously treated with chemotherapy and crizotinib (CRZ). J Clin Oncol. 2015;33(Suppl; abstr 8059).

35. Felip E, Orlov S, Park K, et al. ASCEND-3: A single-arm, open-label, multicenter phase II study of ceritinib in ALKi-naïve adult patients (pts) with ALK-rearranged (ALK+) non-small cell lung cancer (NSCLC). J Clin Oncol. 2015;33(Suppl; abstr 8060).

36. Eisenhauer EA, Therasse P, Bogaerts J, et al. New response evaluation criteria in solid tumours: revised RECIST guideline (version 1.1). Eur J Cancer. 2009;45:228-247.

37. Rangachari D, Yamaguchi N, VanderLaan PA, et al. Brain metastases in patients with EGFR-mutated or ALK-rearranged non-small-cell lung cancers. Lung Cancer. 2015;88(1):108-111.

38. Costa DB, Shaw AT, Ou S-HI, et al. Clinical experience with crizotinib in patients with advanced ALK-rearranged non-small-cell lung cancer and brain metastases. J Clin Oncol. 2015;33(17):1881-1888.

39. Shaw A, Mehra R, Tan DSW, et al. BM-32Ceritinib (LDK378) for treatment of patients with ALK-rearranged (ALK+) non-small cell lung cancer (NSCLC) and brain metastases (BM) in the ASCEND-1 trial. Neuro Onco. 2014; 16(Suppl 9):39.

40. Chugai's ALK Inhibitor “Alecensa ${ }^{\circledR}$ ” Trial Stopped Early for Benefit. [press release]. Tokyo, Japan. Business Wire; 2016 [February 10]. Available from: http:/www.businesswire.com/news/home/20160210005605/ en/Chugais-ALK-Inhibitor-Alecensa\%C2\%AE-Trial-Stopped-Early. Accessed April 28, 2016.

41. Sakamoto H, Tsukaguchi T, Hiroshima S, et al. CH5424802, a selective ALK inhibitor capable of blocking the resistant gatekeeper mutant. Cancer Cell. 2011;19(5):679-690. 
Therapeutics and Clinical Risk Management

Dovepress

\section{Publish your work in this journal}

Therapeutics and Clinical Risk Management is an international, peerreviewed journal of clinical therapeutics and risk management, focusing on concise rapid reporting of clinical studies in all therapeutic areas outcomes, safety, and programs for the effective, safe, and sustained use of medicines. This journal is indexed on PubMed Central, CAS,
EMBase, Scopus and the Elsevier Bibliographic databases. The manuscript management system is completely online and includes a very quick and fair peer-review system, which is all easy to use. Visit http://www.dovepress.com/testimonials.php to read real quotes from published authors.

Submit your manuscript here: http://www.dovepress.com/therapeutics-and-clinical-risk-management-journal 